\title{
ARTICLE \\ Piezoelectricity of ZnO Films Prepared by Sol-Gel Method ${ }^{\dagger}$
}

\author{
Ke-ming Zhang ${ }^{a, b}$, Ya-pu Zhao ${ }^{a *}$, Fa-quan He ${ }^{a}$, Dong-qing Liu ${ }^{b}$ \\ a. State Key Laboratory of Nonlinear Mechanics, Institute of Mechanics, Chinese Academy of Sciences, \\ Beijing 100080, China; \\ b. Department of Mathematics and Mechanics, Beijing University of Science and Technology, Beijing \\ 100083, China
}

(Dated: Received on August 30, 2007; Accepted on October 11, 2007)

\begin{abstract}
$\mathrm{ZnO}$ piezoelectric thin films were prepared on crystal substrate $\mathrm{Si}(111)$ by sol-gel technology, then characterized by scanning electron microscopy, X-ray diffraction and atomic force microscopy (AFM). The ZnO films characterized by X-ray diffraction are highly oriented in (002) direction with the growing of the film thickness. The morphologies, roughness and grain size of $\mathrm{ZnO}$ film investigated by AFM show that roughness and grain size of $\mathrm{ZnO}$ piezoelectric films decrease with the increase of the film thickness. The roughness dimension is $2.188-0.914 \mathrm{~nm}$. The piezoelectric coefficient $d_{33}$ was investigated with a piezo-response force microscope (PFM). The results show that the piezoelectric coefficient increases with the increase of thickness and (002) orientation. When the force reference is close to surface roughness of the films, the piezoelectric coefficient measured is inaccurate and fluctuates in a large range, but when the force reference is big, the piezoelectric coefficient $d_{33}$ changes little and ultimately keeps constant at a low frequency.
\end{abstract}

Key words: $\mathrm{ZnO}$ thin films, Piezoelectric coefficient, Piezo-response force microscope, Sol-gel, Surface roughness

\section{INTRODUCTION}

$\mathrm{ZnO}$ piezoelectric thin film is a very useful multifunction semiconductive material and is widely used in piezoelectric, transparent conducting electrodes, optoelectric devices, and gas sensors because of their excellent characteristics. It is also attractive for high frequency surface acoustic wave device application [1]. $\mathrm{ZnO}$ is a novel wide direct-gap semiconductor with an energy gap of $3.3 \mathrm{eV}$ and a high free exciton binding energy of $60 \mathrm{meV}$ at room temperature which has great usages in information age. There has been great progress in the science of piezoelectricity and its applications have mushroomed in many fields, such as medicine, military affairs, computers and telecommunications. $\mathrm{ZnO}$ has recently become a very fashionable material due to its applications generated by particular properties [2]. Its high piezoelectricity coefficient and electromechanical coupling factor have led to substantial research into the origin of its strong piezoelectricity and applications. Piezoelectric characterization of individual $\mathrm{ZnO}$ nanobelt has been investigated by piezo-response force microscope (PFM) [3]. In that work, the effective piezoelectric coefficient $d_{33}$ of $\mathrm{ZnO}$ nanobelt was found to be frequency dependent and much larger than that of the bulk (0001) $\mathrm{ZnO}$, which support the application of $\mathrm{ZnO}$ nanobelts as nanosensors and nanoactuators.

\footnotetext{
$\dagger$ Part of the special issue from "The 6th China International Conference on Nanoscience and Technology, Chengdu (2007)"

* Author to whom correspondence should be addressed. E-mail: yzhao@imech.ac.cn, Tel: +86-10-82543932.
}

The piezoelectric coefficient and resistivity of sputtered $\mathrm{ZnO}$ films undoped and doped with $\mathrm{Cu}, \mathrm{Ni}, \mathrm{Co}$, and $\mathrm{Fe}$ have been investigated and the results indicated that $\mathrm{Cu}$ dopant can enhance the $c$-axis orientation and the piezoelectric coefficient $\left(d_{33}\right)$ which is promising to fabricate the $\mathrm{ZnO}$ films doped with $\mathrm{Cu}$ for $\mathrm{SAW}$ device applications [4]. In general, $\mathrm{ZnO}$ films prepared by many methods did not have good performance and there would always be too large a roughness or big particles on the film surface. Especially, roughness on the films surface can play an important role in micro/nano sensors or actuators. Recently in the study of $\mathrm{ZnO}$ piezoelectric films, many researchers have been concerned with the preparation techniques and characterization, but few reports discuss the effect of roughness on the piezoelectric films. In this work, the piezoelectric characterization and the effect of different roughnesses on $\mathrm{ZnO}$ piezoelectric films were discussed in detail.

Recently various $\mathrm{ZnO}$ semiconductor nanostructures have been fabricated, such as an oriented structure of $\mathrm{ZnO}$ nanorods and $\mathrm{ZnO}$ nanowires [5]. $\mathrm{ZnO}$ thin films can be obtained by different methods (sol-gel technique, chemical vapour deposition (CVD), pulse laser deposition (PLD), spray pyrolysis, magnetron sputtering, etc.). Sol-gel technique have many advantages in preparing $\mathrm{ZnO}$ films, such as strong $c$-axis orientation, ease of compositional modifications, large films, simplicity of working principle, low cost, and low annealing temperature. The sol-gel method is therefore prevalent today and ideal for exploratory research.

When an electric field is applied to a piezoelectric material, it strains due to the converse piezoelectric effect. In ideal PFM piezoelectric measurement, an electric field is applied to a piezoelectric material, and the 
tip accurately follows the piezoelectric motion. Since the piezoelectric constant represents the linear relation between the strain and applied field, the piezoelectric motion will also be of the frequency at which the piezoelectric is driven by a voltage. These small piezoelectric displacements can be measured in several techniques, including PFM. Piezoelectric measurements of $\mathrm{ZnO}$ piezoelectric films were performed in contact mode with a PFM. PFM has become a useful characterization technique and has been described in several books [6-8] and in the literatures $[3,4]$. The PFM technique has the advantage of being able to measure the piezoelectric effect at single points on a sample. In this configuration, it is more difficult to accurately determine the electric field, but the measurements can be performed readily and directly upon the $\mathrm{ZnO}$ piezoelectric thin film. The PFM technique measuring the absolute magnitude of the effective longitudinal $d_{33}$ is based on the detection of vibrations of $\mathrm{ZnO}$ piezoelectric films and a laser and position sensitive photodetector (PSPD) combination which can detect the cantilever deflection. The conductive tip that supplies current to the film and also measures the piezoelectric motion due to the inverse piezoelectric effect is made by coating $\mathrm{Rh}$ and the tip radius is about $10 \mathrm{~nm}$. The input signal is in the range of $1-5 \mathrm{~V}$, and the vertical deflection signal $A_{0}$ of the cantilever is recorded by a lock-in amplifier. The slope of the amplitude versus the input signal $U_{0}$ gives $\mathrm{d}_{33}$.

\section{EXPERIMENTS}

The piezoelectricity of $\mathrm{ZnO}$ films prepared by solgel with different thickness and roughness is a key characteristic for the piezoelectric measurement. The $\mathrm{ZnO}$ piezoelectric films were deposited on $\mathrm{Si}(111)$ wafer substrates by the sol-gel process. Sol was prepared by dissolving zinc acetate (ZnAc) (99\% purity) in 2methoxyethanol and MEA (1:1:1 in mol). MEA and 2-methoxyethanol were used as stabilizer and solvent, respectively. The mixture was confected to $0.35 \mathrm{~mol} / \mathrm{L}$ and stirred ultrasonically. A magnetic stirrer was used at $55{ }^{\circ} \mathrm{C}$ for $60 \mathrm{~min}$ till a clear solution was formed which was found to be stable and transparent with no precipitate or turbidity after cooling to room temperature. The coating was made $48 \mathrm{~h}$ after the sol was prepared in the room temperature in order to make it more glutinous. The single-crystalline $\mathrm{Si}(111)$ substrates were degreased in the mixture of acetone and tetrachloride carbon (1:1 in volume) and stirred ultrasonically for $30 \mathrm{~min}$, and then washed 10 times by distilled water, and in the end dehydrated by alcohol many times. Before coating, substrates were dipped in HF solution (5\%) for $10 \mathrm{~s}$, washed by distilled water completely, and then dehydrated by alcohol standby. The sol was dripped onto $\mathrm{Si}(111)$ substrate and then the substrate was spin-coated with the sol at a speed of $3200 \mathrm{r} / \mathrm{min}$ for $40 \mathrm{~s}$ in air. After each coating on the

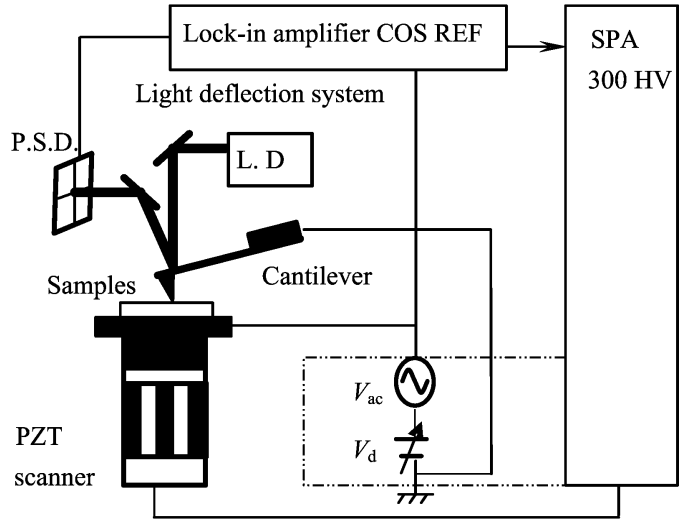

FIG. 1 Schematic diagram of experiments for piezoelectric measurements.

substrate, the substrate coated with $\mathrm{ZnO}$ film layer was dried at $100{ }^{\circ} \mathrm{C}$ for 5 min over a hot plate in air. Then the deposited $\mathrm{ZnO}$ film was annealed in air at a temperature of $500{ }^{\circ} \mathrm{C}$ for $1 \mathrm{~h}$ to complete one cycle of crystallization of the film. This process of coating and subsequent heat treatment was repeated seven times in order to get thicker $\mathrm{ZnO}$ films.

The thickness of the $\mathrm{ZnO}$ film was built up by increasing the number of coatings (1-7 coating cycles). The thickness of each $\mathrm{ZnO}$ film was measured by a scanning electronic microscope (SEM) which was also used to illustrate the formation of crystallites on the $\mathrm{ZnO}$ film surface. X-ray diffraction (XRD) was used for structural characterization and phase identification of $\mathrm{ZnO}$ films using $\mathrm{Cu} \mathrm{K} \alpha$ radiation operating at $40 \mathrm{kV}$, $150 \mathrm{~mA}$. The surface morphology and roughness of the deposited $\mathrm{ZnO}$ films was investigated using AFM. Piezoelectric coefficient $d_{33}$ measurements of the series of films with different roughness were performed with a PFM (Seiko SPA300HV) in contact mode. A schematic diagram of piezoelectric measurement system is shown in Fig.1. The function generator drives the $\mathrm{ZnO}$ thin film piezoelectric capacitor, and in the experiments the frequency varies from $200 \mathrm{~Hz}$ up to $1 \mathrm{kHz}$ which is beyond most environmental noise frequencies and well below the tip resonances. The peak-to-peak potential varies up to $5 \mathrm{~V}$. The frequency and the applied voltage are stepped through computer control, and the lockin amplifier records the PFM tip displacement at the frequency. From the slope of the resulting displacement vs. applied voltage plot, we can calculate the piezoelectric constant. Measurements were performed on $\mathrm{ZnO}$ piezoelectric thin films with different thickness, frequency and voltage.

\section{RESULTS AND DISCUSSION}

\section{A. Microstructure of $\mathrm{ZnO}$ piezoelectric films}

The structural properties and the cross-section of $\mathrm{ZnO}$ films were investigated by XRD and SEM as shown 


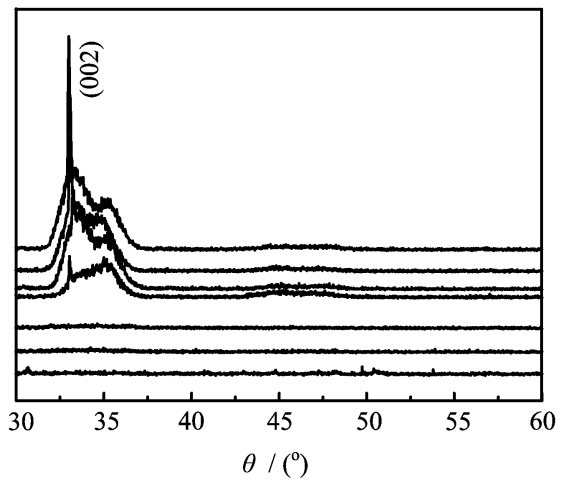

FIG. 2 XRD pattern of the thin $\mathrm{ZnO}$ films with seven different thickness of $20,30,53,62,70,80$, and $88 \mathrm{~nm}$ from bottom to top.

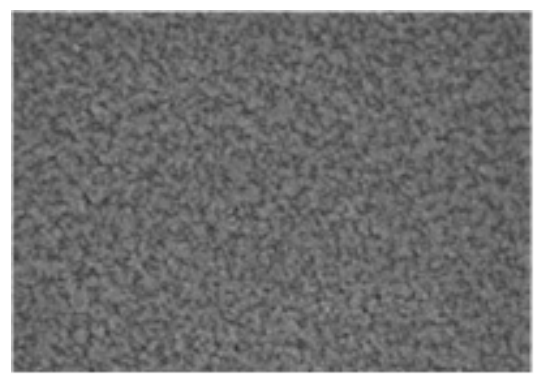

(a)

$500 \mathrm{~nm}$

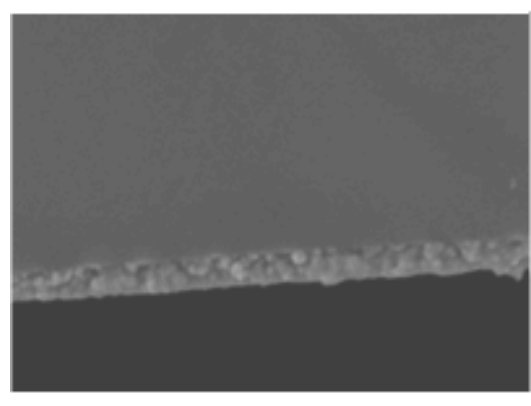

(b)

$500 \mathrm{~nm}$

FIG. 3 Surface SEM image (a) and cross-section SEM image (b) of the deposited $\mathrm{ZnO}$ film with thickness of $88 \mathrm{~nm}$.

in Figs. 2, 3, and 4. Figure 2 shows the XRD patterns of $\mathrm{ZnO}$ piezoelectric films with thickness of $20,30,53$, $62,70,80$, and $88 \mathrm{~nm}$, respectively. From the figure we can see that as the film thickness increases from $20 \mathrm{~nm}$ to $88 \mathrm{~nm}$ which is polycrystalline with wurtzite structure, the intensity of (002) peak ( $c$-axis orientation) increases, which implies an improvement in the crystalline quality of the film. The SEM micrographs of ZnO films with seven different thicknesses showed uniform tightly packed grains whose size decreases with the increase in thickness. The surface and cross-sectional SEM image of film with a thickness of $80 \mathrm{~nm}$ grown on $\mathrm{Si}(111)$ by sol-gel was shown in Fig.3.

In Fig.2, the (002) peak of thin films moves to a small angle and are fat compared with thick $\mathrm{ZnO}$ films

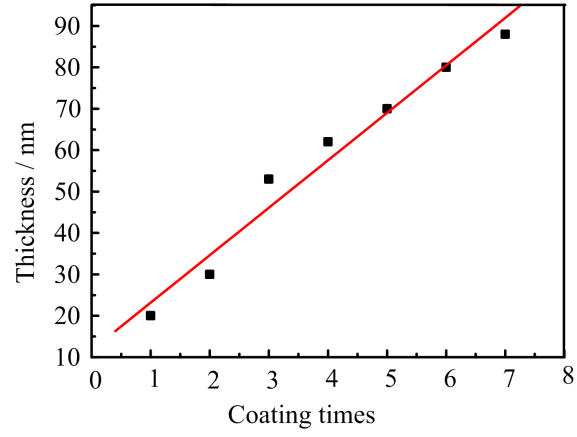

FIG. 4 The thickness of the films at different coating times.

$(>100 \mathrm{~nm})$ in the literature. The reason may be that the films are very thin and in the $\mathrm{ZnO}$ thin films stress exists after annealing. The lattice parameters were calculated using the observed values of $2 \theta$ for (002) plane and (101) plane and the $d$-values (inter-planar spacing) for the hexagonal structure, which is given by

$$
\frac{1}{d^{2}}=\frac{4\left(h^{2}+h k+k^{2}\right)}{3 a^{2}}+\frac{l^{2}}{c^{2}}
$$

where $d$ is inter-planer spacing, $a$ and $c$ are base vectors of hexangular lattice, $h, k, l$ are exponential of crystal surface. As to $\mathrm{ZnO}(002), h=k=0, l=2$, hence $c=2 d$. Since $2 \delta \sin \theta=n l$, when $n=1$, the lattice parameter of $\mathrm{ZnO}$ film was calculated using the observed values of $2 \theta$ for (002) plane, which is given by

$$
c=\frac{\lambda}{2 \sin \theta} \sqrt{\frac{4}{3(a / c)^{2}}\left(h^{2}+h k+k^{2}\right)+l^{2}}
$$

where $\lambda, \theta$ are wavelength of X-ray $(0.15418 \mathrm{~nm})$ and angle of diffraction, respectively. The lattice parameter of $\mathrm{ZnO}$ film calculated is a little bigger than that of $\mathrm{ZnO}$ bulk $(0.521 \mathrm{~nm})$. Because tensile stress exists in the $\mathrm{ZnO}$ film at $c$-axis orientation, which makes the lattice parameter become bigger and diffractive apex move to a small angle.

According to Wu et al. [9] and Chen et al. [10], a film with a smooth surface which is well-oriented by X-ray analysis exhibits a high effective SAW coupling factor, while a rough surface will enlarge the insertion loss of the SAW device. For $\mathrm{ZnO}$ piezoelectric film, the surface smoothness is a critical characteristic of piezoelectric material for the fabrication of low loss piezoelectric devices and high frequency surface acoustic wave device application.

To study the effect of roughness against piezoelectric coefficient $d_{33}$, the samples prepared by sol-gel were investigated by AFM. The results indicated that the $\mathrm{ZnO}$ films have a smooth surface with the roughness from $2.188 \mathrm{~nm}$ to $0.914 \mathrm{~nm}$. Figure 5 illustrates the typical AFM morphologies of $\mathrm{ZnO}$ films grown on $\mathrm{Si}(111)$ with different thickness. As shown in the figure, the surface of the $\mathrm{ZnO}$ thicker film was smoother than the thinner. 

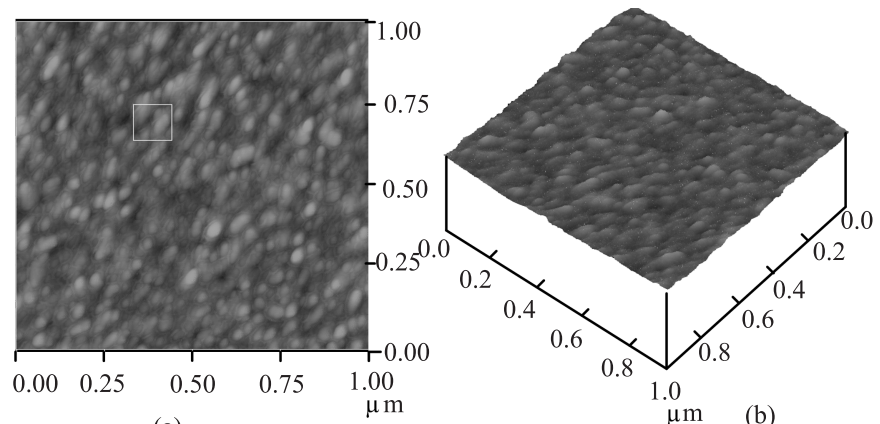

(a)

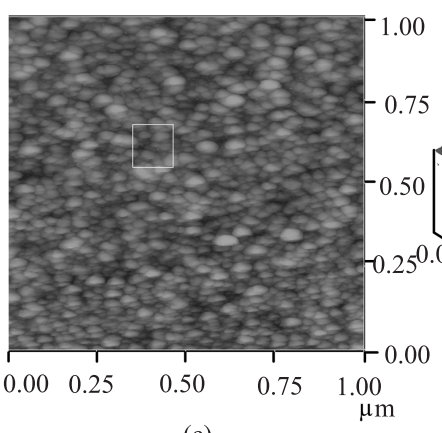

(c)

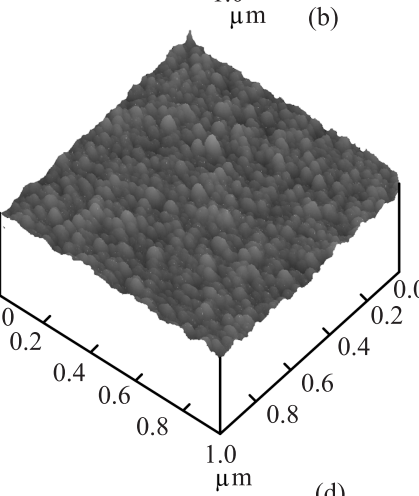

(d)
FIG. 5 (a) and (c) AFM images and roughness analysis of the $\mathrm{ZnO}$ film with thickness of 20 and $30 \mathrm{~nm}$, respectively; (b) and (d) three-dimensional image of $\mathrm{ZnO}$ films.

The average surface roughness $\left(R_{a}\right)$ of $\mathrm{ZnO}$ films at different thickness of $20,30,53,62,70,80$, and $88 \mathrm{~nm}$ was $2.188,1.892,1.891,1.291,1.245,1.015$, and 0.914 , respectively. The grain becomes denser with the increase in thickness.

\section{B. Piezoelectric properties of $\mathrm{ZnO}$ films}

PFM is becoming a standard method for the study of piezoelectric phenomena $[3,4]$. The piezoelectric properties of $\mathrm{ZnO}$ films with smooth and rough surfaces were measured using the PFM technique in contact mode. The sample first was located on the sample platform with silver cataplasm, then the sample with silver cataplasm was heated for $60 \mathrm{~min}$ in a heater at a temperature of $100{ }^{\circ} \mathrm{C}$. A conductive tip coated with $\mathrm{Rh}$ was used as a top electrode $(0 \mathrm{~V})$ which can obtain the $z$-deflection signal.

An ac signal is applied between the grounded tip electrode and the substrate electrode. When the electric field is applied to a piezoelectric $\mathrm{ZnO}$ film, it strains due to the converse piezoelectric effect. The tip perpendicular displacement is sensed by the photo-sensitive detector and its output signal is split into amplitude and phase by a lock-in amplifier. The ac signal was employed with a frequency of $0.5 \mathrm{kHz}$, and the peak-topeak potential varied up to $5 \mathrm{~V}$. The $0.5 \mathrm{kHz}$ frequency is below the tip resonances and above most environmental noise whose frequency range is $20-200 \mathrm{~Hz}$. In the

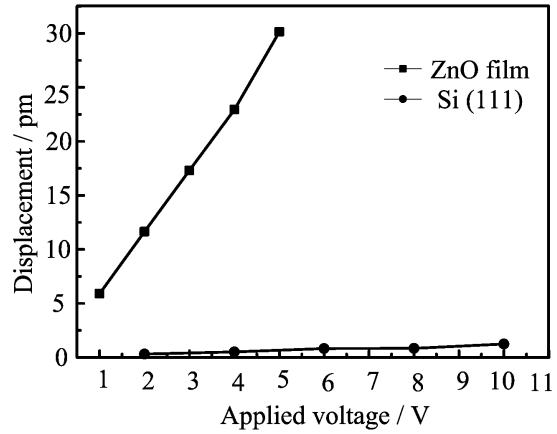

FIG. 6 Piezoelectric measurements of thin film $\mathrm{ZnO}$ with thickness of $20 \mathrm{~nm}$ and single-crystalline Si (111). Piezoelectric coefficient of the samples for the measurements were $d_{33}=5.81 \mathrm{pm} / \mathrm{V}$ and $d=0.26 \mathrm{pm} / \mathrm{V}$ while the experimental noise level was $0.5 \mathrm{pm} / \mathrm{V}$.

piezoelectric measurement experiments, we changed the frequency from $200 \mathrm{~Hz}$ to $1 \mathrm{kHz}$ and amplitude of voltage which is stepped through computer control. Also, we changed the depth of the tip's penetration into the $\mathrm{ZnO}$ films so as to research the relation between the piezoelectric coefficient and roughness. The displacement of cantilever at the frequency $\omega$ and amplitude of voltage $A$ is recorded by a lock-in amplifier. The piezoelectric coefficient $d_{33}$ can be calculated from the slope of the resulting amplitude of displacement vs. that of applied voltage plot, which are both peak-to-peak values of displacement and applied voltage, respectively. The piezoelectric coefficient $d_{33}$ is calculated by $[3,11]$

$$
d_{33}=A_{0} / U_{0}
$$

where $A_{0}$ is vibration amplitude, $U_{0}$ is the amplitude of the testing ac voltage.

Piezoelectric measurements were performed on seven types of thin $\mathrm{ZnO}$ films with seven types of thickness and roughness, and also on Si substrate with no film. Figure 6 shows piezoelectric measurements of $\mathrm{ZnO}$ thin film (20 nm in thickness) and single-crystalline $\mathrm{Si}(111)$. Piezoelectric constants for the measurements were $d_{33}=5.81 \mathrm{pm} / \mathrm{V}$ and $d=0.26 \mathrm{pm} / \mathrm{V}$. The singlecrystalline Si measurement is at our experimental noise level $(<0.5 \mathrm{pm} / \mathrm{V})$. In all the piezoelectric measurement, the interaction between the tip and electric field present was ignored [11].

Figure 7 is piezoelectric measurements of varies thickness $\mathrm{ZnO}$ thin films. As the figure shown, the piezoelectric coefficient $d_{33}$ is size-dependent from 5.81-28.7 pm/V, which is bigger than that of the bulk (0001) $\mathrm{ZnO}(9.93 \mathrm{pm} / \mathrm{V})$ in the strong $c$-axis orientation. From the result of measurements, when the $\mathrm{ZnO}$ thin films are not strong $c$-axis orientation, the piezoelectric coefficient $d_{33}$ is close to that of the bulk. When the films are approaching strong $c$-axis orientation, $d_{33}$ is increasing rapidly. Therefore, the piezoelectric coefficient $d_{33}$ increases with increasing thickness size. 

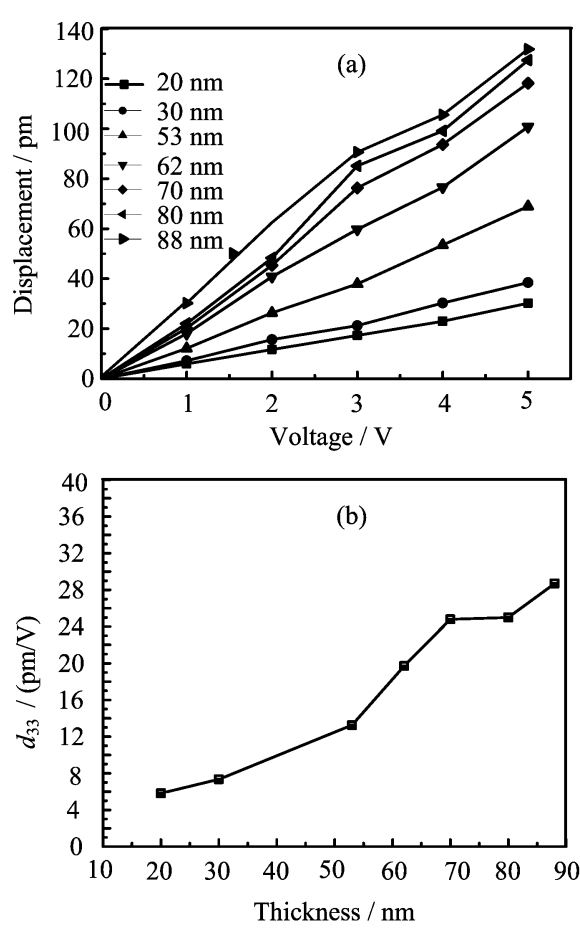

FIG. 7 (a) Piezoelectric measurements of seven $\mathrm{ZnO}$ thin piezoelectric films: size-dependence of piezoelectric coefficient $d_{33}$. Each value in the figure is the average of 10 points measurement and each point is performed at an applied voltage amplitude from $1 \mathrm{~V}$ to $5 \mathrm{~V}$ with frequency of $500 \mathrm{~Hz}$. (b) the trend of $d_{33}$ intuitionisticly.

Since the $\mathrm{ZnO}$ thin films is not absolutely smooth which were very rough against the thickness in nanoscale. Figure 8 shows that when the thickness of the film was $88 \mathrm{~nm}$, the piezoelectric constant $d_{33}$ fluctuated greatly owing to the loose contact between the tip and the film surface which were both vibrating with a frequency $\omega$. Force Reference (Force Ref.) must be negative, the approach will not work properly if it is positive. The value of the $x$-coordinate in the Fig. 8 is the absolute value of the Force Ref. The AFM tip radius is about $25 \mathrm{~nm}$ while the top electrode is the small area where the tip was in contact with the thin film. To calculate the depth of the tip's penetration into the film, we use the Hertz model (Fig.9), in which the tip is regarded as a sphere and the film as an infinity plane. In the modeling, the radius and the elastic modulus of the tip are $R$ and $E_{1}$, respectively; the elastic modulus of $\mathrm{ZnO}$ film is $E_{2}$; the Poisson's ratios of the tip and the film are $\lambda_{1}$ and $\lambda_{2}$, respectively. According to Hertz contact theory, the radius of contact area is:

$$
\begin{gathered}
a^{3}=R P / K \\
K=\frac{4}{3}\left[\frac{\left(1-\lambda_{1}^{2}\right)}{E_{1}}+\frac{\left(1-\lambda_{2}^{2}\right)}{E_{2}}\right]^{-1}
\end{gathered}
$$

The depth of compression is calculated by formula below $\left(E_{1}=60 \mathrm{GPa}, \lambda_{1}=0.3 ; E_{2}=300 \mathrm{GPa}, \lambda_{2}=0.24\right.$;
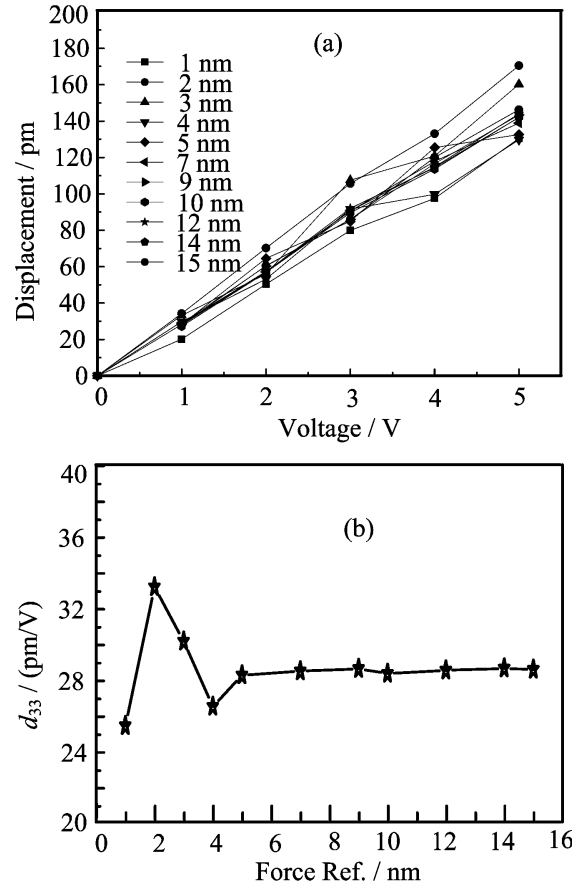

FIG. 8 (a)Piezoelectric measurements of the thin $\mathrm{ZnO}$ film (88 nm thick) with Force Ref. from 1-15 nm. The piezoelectric constant $d_{33}$ fluctuates greatly owing to the loose contact between the tip and the film surface which were both vibrating with a frequency. (b) shows the trend of $d_{33}$ intuitionisticly.

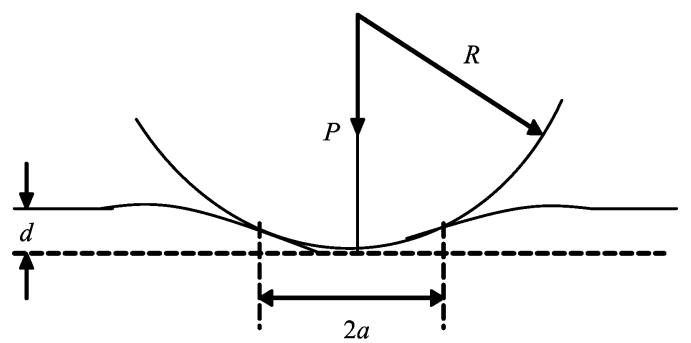

FIG. 9 The Hertz model.

$R=10 \mathrm{~nm} ; \mathrm{P} \approx 20 \mathrm{nN})$.

$$
\delta=a^{2} / R
$$

When the Force Ref. reaches $4 \mathrm{~nm}$, the depth of the tip into the film is about $\delta=1.335 \mathrm{~nm}$, which is a little bigger than the roughness $(0.914 \mathrm{~nm})$ of the film. As the depth of the tip into the film is the same magnitude as the roughness of the film, the effective coefficient does not fluctuate greatly. Thus, microscopic variation and the approach of the piezoelectric film will affect the electrode quality. As to (002) $\mathrm{ZnO}$ nanobelt, Zhao et al. found that the effective piezoelectric coefficient $d_{33}$ is frequency dependent and is decreasing with increasing frequency which is high [3]. Here, piezoelectric measurements were performed at a low frequency $(200 \mathrm{~Hz}-$ $1 \mathrm{kHz}$ ) on $\mathrm{ZnO}$ thin film. In generally, the frequency of 

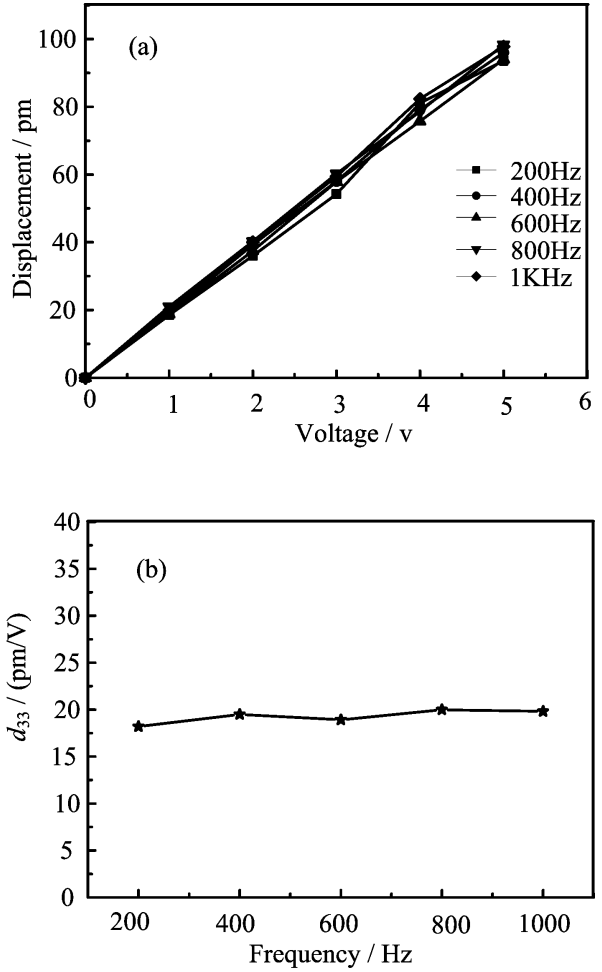

FIG. 10 (a) The ZnO film (62 nm thick) piezoelectric measurements at a low frequency from $200 \mathrm{~Hz}$ to $1 \mathrm{kHz}$. The $d_{33}$ changes little and ultimately keeps constant. (b) The trend of $d_{33}$.

noise is below $200 \mathrm{~Hz}$, and $1 \mathrm{kHz}$ is the upper limit frequency put by the AFM. Figure 10 shows that at a low frequency, the piezoelectric coefficient of $\mathrm{ZnO}$ thin film is not frequency dependent and ultimately keeps constant which does not conflict with the result of Zhao et al. measured at a high frequency $(\sim 100 \mathrm{kHz})$.

\section{CONCLUSION}

A series of $\mathrm{ZnO}$ piezoelectric thin films on singlecrystalline $\mathrm{Si}(111)$ substrate were prepared by sol-gel technique. The characterization of the films was investigated by AFM, SEM and XRD. The piezoelectric measurements were performed using PFM. When the thickness of the $\mathrm{ZnO}$ films is below $100 \mathrm{~nm}$, the piezoelectric coefficient increases with the increasing thickness due to the strong $c$-axis orientation. When the Force Ref. is big enough to ensure the contact between the tip and the film, the value measured is trustworthy, otherwise the value of piezoelectric coefficient fluctuates greatly. The results of piezoelectric measurements reveal that the piezoelectric coefficient of $\mathrm{ZnO}$ film is not frequency dependent at a low frequency, but is high frequency dependent.

\section{ACKNOWLEDGMENTS}

This work was supported by the National Basic Research Program of China (No.2007CB310500). Helpful discussion with Dr. Hui-ling Li is gratefully acknowledged.

[1] K. Koike, H. Tanaka, and H. Ieki, Jpn. J. Appl. Phys. 34, 2678 (1995).

[2] Z. L. Wang and J. H. Song, Science 312, 242 (2006).

[3] M. H. Zhao, Z. L. Wang, and S. X. Mao, Nano Lett. 4, 587 (2004).

[4] X. B. Wang, C. Song, D. M. Li, K. W. Geng, F. Zeng, and F. Pan, Appl. Surf. Sci. 253, 1639 (2006).

[5] F. Q. He and Y. P. Zhao, Appl. Phys. Lett. 88, 193113 (2006).

[6] R. Wiesendanger. Scanning Probe Microscopy and Spectroscopy, Cambridge: Cambridge University Press, (1994).

[7] D. Sarid. Scanning Force Microscopy. NY: Oxford University Press, (1991).

[8] R. Howland and L. Benatar, A Practical Guide of Scanning Probe Microscopy, Sunnyvale: Park Scientific Instruments (1993).

[9] M. S. Wu, W. C. Shih, and W. H. Tsai, J. Phys. D: Appl. Phys. 31, 943 (1998).

[10] J. J. Chen, Y. Gao, F. Zeng, D. M. Li, and F. Pan, Appl. Surf. Sci. 223, 318 (2004).

[11] J. A. Christman, R. R. Woolcott, A. I. Kingon, and R. J. Nemanich, Appl. Phys. Lett. 73, 3851 (1998). 